\title{
Ranolazine Reduces Patient-Reported Angina Severity and Frequency and Improves Quality of Life in Selected Patients with Chronic Angina
}

\author{
Joseph B. Muhlestein · Sharon Grehan
}

Published online: 29 August 2013

(C) The Author(s) 2013. This article is published with open access at Springerlink.com

\begin{abstract}
Background Chronic stable angina negatively affects quality of life (QoL). American College of Cardiology/ American Heart Association guidelines highlight maintaining/restoring a level of activity, functional capacity, and QoL that is satisfactory to the patient as an objective of treatment, and further define the treatment goal for most patients as maximizing survival and achieving prompt and complete (or near-complete) elimination of angina with a return to normal activities.

Objective To assess self-reported angina severity, frequency, and QoL in patients with chronic stable angina who had not undergone revascularization and who were prescribed and remained on ranolazine.

Methods Patients $(N=92)$ answered a survey evaluating their perceptions of angina prior to ranolazine initiation (based on recall of previous experience) and during ranolazine treatment. Change in QoL was assessed using the Patient Global Impression of Change scale.

Results Most respondents were female $(64 \%)$ and had taken ranolazine for $\geq 6$ months $(89 \%)$; mean age was 64 years. The majority of respondents selected higher scores for angina severity before ranolazine treatment (54\%), and lower scores for severity while on ranolazine $(68 \%)$. Most respondents reported experiencing $\geq 1$ angina attack/week before ranolazine treatment $(82 \%)$ and $<1$ attack/week while on ranolazine $(73 \%)$. The effect of
\end{abstract}

\footnotetext{
J. B. Muhlestein $(\square)$

Intermountain Medical Center, 5121 S. Cottonwood Street, Murray, UT 84107, USA

e-mail: brent.muhlestein@imail.org

S. Grehan

Gilead Sciences, Inc, Foster City, CA, USA
}

angina on daily activities was less while taking ranolazine than before ranolazine treatment; 52 and $8 \%$ of respondents, respectively, reported significant impact, and 12 and $67 \%$, respectively, reported little/no impact. Most respondents reported noticeably improved angina-related QoL since starting ranolazine (79\%).

Conclusion Patients who maintained ranolazine treatment for durations ranging from $<6$ months to $>4$ years reported substantial improvements in angina severity, frequency, and QoL.

\section{Introduction}

Coronary heart disease (CHD) is the most common form of heart disease in the United States (US), affecting an estimated 15.4 million adults aged $\geq 20$ years $(6.4 \%)$ [1]. In 2009, almost 400,000 deaths were attributed to CHD, and each year approximately 635,000 individuals will have a primary coronary attack. An estimated 7.8 million adults aged $\geq 20$ years $(3.2 \%)$ in the US experience angina pectoris, a recurrent and debilitating chest pain, which is an underlying symptom of CHD [1]. Chronic stable angina is diagnosed in approximately 500,000 individuals aged $\geq 45$ years annually, and is a negative predictor of quality of life (QoL) in many patients with CHD [1-3]. Angina places a high burden on the US healthcare system, with direct healthcare costs associated with the disease estimated to range from US\$1.9 to US\$75 billion, depending on the definition of angina used [4].

In patients with chronic stable angina, the occurrence of $\geq 1$ episode of angina on a weekly basis is associated with worse QoL and greater physical limitations [5]. Recent American College of Cardiology (ACC)/American Heart Association (AHA) guidelines for the management of stable 
ischemic heart disease highlight maintaining or restoring a level of activity, functional capacity, and QoL that is satisfactory to the patient as one of the objectives of treatment for this patient population. The guidelines define the goal of treatment for most patients as maximizing survival and achieving prompt and complete (or near-complete) elimination of angina with a return to normal activities [6].

Traditional therapies for chronic stable angina include $\beta$-blockers, calcium channel blockers, and long-acting nitrates [6]. For some patients, use of these agents may be limited by key adverse effects of $\beta$-blockers (bradycardia, heart block, hypotension, bronchospasm) and calcium channel blockers (ankle edema, headache, flushing, hypotension), as well as tolerance associated with long-term use of nitrates [7].

The sodium channel inhibitor ranolazine is indicated to treat chronic stable angina and may be used with $\beta$ blockers, calcium channel blockers, and nitrates [8]. When taken in combination with standard doses of $\beta$-blockers or calcium channel blockers, ranolazine improved exercise duration and time to ischemia, and reduced the frequency of angina attacks and nitroglycerin use in patients with severe chronic angina [9].

In a pilot study comparing ranolazine and placebo for 4 weeks each in a crossover fashion in 20 women with angina and evidence of myocardial ischemia but no obstructive coronary artery disease, scores were significantly better for ranolazine on the Seattle Angina Questionnaire (SAQ) subscales assessing physical functioning (91.7 vs. $83.3 ; p=0.046$ ), angina stability (75.0 vs. 50.0 ; $p=0.008$ ), and QoL (75.0 vs. 66.7; $p=0.021)$ [10]. A prospective QoL assessment performed alongside the MERLIN (Metabolic Efficiency with Ranolazine for Less Ischemia in Non-ST-elevation acute coronary syndromes)TIMI 36 trial showed small but statistically significant effects of ranolazine on disease-specific health status and QoL over 12 months' follow-up [11].

Little is known regarding the impact of ranolazine on QoL over longer treatment durations. The present patient survey was designed to evaluate the effect of long-term (up to $>4$ years) ranolazine treatment on self-reported angina severity, frequency, and QoL in patients with chronic angina.

\section{Methods}

A 40-question survey was distributed from 6 April to 10 May 2011, via email and telephone, to a panel of patients currently receiving ranolazine treatment. Patients were invited to participate in the panel through website registration (Ranexa.com and SpeakFromTheHeart.com), by opting-in for research, or via savings program participation. Patients answered screening questions (for which they received honoraria) in order to join the panel; the screening criteria included age $\geq 18$ years; being on ranolazine treatment prescribed by a healthcare professional (not including use of only a sample); and no employment of themselves or any immediate family member by a pharmaceutical manufacturer, medical equipment manufacturer, market research or advertising firm, medical office, clinic, or hospital. Panel members were subsequently invited and opted to participate in the survey.

The survey assessed patients' perceptions of their angina severity and frequency, impact on daily activities, and QoL before initiating ranolazine treatment (based on patientreported recall of their previous experience), and at the time of the survey while on ranolazine. Angina severity was rated using a 7-point Likert scale (where $1=$ extremely mild and $7=$ extremely severe). Respondents classified the frequency of angina attacks as: more than once per day; about once per day; less than once a day, but one or more per week; or less than once a week. The impact of angina on patients' daily activities was also rated using a 7-point Likert scale (where $1=$ not at all and $7=$ a lot). Change in QoL was assessed using the Patient Global Impression of Change (PGIC) scale [12]. Respondents classified changes in activity limitations, symptoms, emotions, and overall QoL related to angina as one of the following categories:

- no change (or condition has got worse);

- almost the same, hardly any change at all;

- a little better, but no noticeable change;

- somewhat better, but the change has not made any real difference;

- moderately better, and a slight but noticeable change;

- better, and a definite improvement that has made a real and worthwhile difference;

- a great deal better, and a considerable improvement that has made all the difference.

In addition, the degree of change experienced was rated using an 11-point Likert scale (where $0=$ much better, $5=$ no change, and $10=$ much worse).

The analysis was limited to respondents who had not undergone revascularization procedures (coronary artery bypass graft or percutaneous coronary intervention [PCI]) to provide a more clear assessment of the effects of ranolazine therapy. Results are presented as percentage of patients.

\section{Results}

\subsection{Survey Participant Demographics}

The survey was distributed to all panel members ( $n=741$; all patients on the panel met the pre-specified screening 
Table 1 Patient demographics

\begin{tabular}{|c|c|}
\hline Characteristics & $\begin{array}{l}\text { Survey respondents } \\
(N=92)\end{array}$ \\
\hline \multicolumn{2}{|l|}{ Sex, $n(\%)$} \\
\hline Female & $59(64)$ \\
\hline Male & $33(36)$ \\
\hline Age, years, mean (range) & $64(35-90)$ \\
\hline Age at angina diagnosis, years, mean (range) & $58(20-88)$ \\
\hline Monthly angina attacks, $n$ (range) & $7(0-60)$ \\
\hline \multicolumn{2}{|l|}{ Duration of ranolazine treatment, $n(\%)$} \\
\hline$<6$ months & $10(11)$ \\
\hline $6-12$ months & $37(40)$ \\
\hline$>1$ to $\leq 2$ years & $29(32)$ \\
\hline$>2$ to $\leq 4$ years & $14(15)$ \\
\hline$>4$ years & $2(2)$ \\
\hline \multicolumn{2}{|l|}{ Time since angina diagnosis, $n(\%)$} \\
\hline$<2$ years & $44(48)$ \\
\hline$\geq 2$ to $<4$ years & $19(21)$ \\
\hline$\geq 4$ to $<6$ years & $6(7)$ \\
\hline$\geq 6$ to $<8$ years & $7(8)$ \\
\hline$\geq 8$ to $<10$ years & $3(3)$ \\
\hline$\geq 10$ years & $13(14)$ \\
\hline \multicolumn{2}{|l|}{ Comorbid CV conditions, $n(\%)$} \\
\hline Any comorbid condition ${ }^{\mathrm{a}}$ & $82(89)$ \\
\hline Atrial fibrillation & $17(18)$ \\
\hline Congestive heart failure & $27(29)$ \\
\hline Diabetes mellitus & $33(36)$ \\
\hline High blood pressure/hypertension & $65(71)$ \\
\hline \multicolumn{2}{|l|}{ Anti-anginal medications, $n(\%)$} \\
\hline Any anti-angina medication ${ }^{\mathrm{b}}$ & $70(76)$ \\
\hline Atorvastatin & $25(27)$ \\
\hline Clonazepam & $4(4)$ \\
\hline Digoxin & $4(4)$ \\
\hline Nitroglycerin & $59(64)$ \\
\hline Verapamil & $6(7)$ \\
\hline Warfarin & $1(1)$ \\
\hline
\end{tabular}

${ }^{a}$ Includes atrial fibrillation, congestive heart failure, and high blood pressure/hypertension

b In addition to ranolazine; includes atorvastatin, warfarin, digoxin, clonazepam, and nitroglycerin

criteria), and 399 patients (54\%) completed the survey. The results from 92 panel members who answered the survey and had not undergone revascularization are presented herein. The majority ( $59 \%$ ) completed the survey by phone, the rest via email. Table 1 summarizes the baseline characteristics of the population, their comorbid cardiovascular conditions, and any additional anti-angina medications used at the time of the survey. The majority of respondents were female $(64 \%)$, and the mean age was 64 years. At the time of the survey, approximately half of the respondents had been diagnosed with angina for $\geq 2$ years $(52 \%)$, and most respondents had been taking ranolazine for $\geq 6$ months ( $89 \%$ ). Almost $90 \%$ of patients surveyed had a cardiovascular condition in addition to angina, and approximately three-quarters of the population received ranolazine therapy plus an additional anti-angina medication.

\subsection{Angina Severity and Frequency}

Angina severity before ranolazine treatment was scored as 6 or 7 by $54.4 \%$ of respondents and as 1 or 2 by only $5.4 \%$ of respondents; severity ratings after ranolazine were 6 or 7 in $2.2 \%$ of respondents and 1 or 2 in $68.5 \%$ of respondents (Fig. 1).

The proportion of respondents reporting angina frequencies of $<1$ attack per day but $\geq 1$ attack per week, about 1 attack per day, and $>1$ attack per day were lower after ranolazine therapy than before ranolazine, while the proportion reporting the least frequent category of $<1$ attack per week was higher after ranolazine (Fig. 2).

\subsection{Impact of Angina on Daily Activities and Quality of Life}

When respondents were asked to rate the impact of angina on their daily activities, $52.1 \%$ of respondents reported that angina had a significant impact on their daily activities (score of 6 or 7) before treatment with ranolazine; only $7.6 \%$ of respondents reported a significant impact after ranolazine (Fig. 3). The proportion of respondents reporting little/no impact of angina on daily activities (score of 1 or 2) increased from $11.9 \%$ before ranolazine to $67.4 \%$ after ranolazine.

As assessed by the PGIC scale, a majority of respondents (79.4\%) reported a noticeable improvement (moderately better, better, or a great deal better) in QoL since initiating ranolazine therapy (Fig. 4). The degree of change in QoL revealed that $89.1 \%$ of patients reported a score of $<5$.

\section{Discussion}

In this patient survey, respondents with chronic angina who did not have a history of revascularization reported substantial improvement in QoL, angina frequency, and angina severity after initiating therapy with ranolazine. These improvements represent key treatment goals established by ACC/AHA guidelines for patients with chronic stable angina.

Chronic stable angina can have a significant negative impact on daily activities and QoL of patients with CHD [13]. Invasive procedures such as PCI, coronary artery bypass grafting, and stenting have been shown to improve 
Fig. 1 Patient-reported angina severity before and after taking ranolazine. $N=92$ respondents. Angina severity was rated using a 7-point Likert scale (where $1=$ extremely mild and $7=$ extremely severe)

Fig. 2 Patient-reported frequency of angina attacks before and after taking ranolazine. $N=92$ respondents


Angina Frequency

QoL in patients with severe angina [14, 15]. However, many patients with stable ischemic heart disease may benefit from medical therapy [16]. Interestingly, among patients with stable angina in the RITA-2 (Second Randomized Intervention Treatment of Angina) and COURAGE (Clinical Outcomes Utilizing Revascularization and Aggressive druG Evaluations) trials, early superiority of PCI over medical therapy in improving QoL had attenuated by 3 years, although this observation may be attributable in part to patients assigned to medical therapy subsequently undergoing invasive treatment $[15,17]$. In COURAGE, patients with more severe and more frequent angina were found to gain the greatest benefit from PCI [15].

Ranolazine can be used as initial anti-anginal therapy (particularly in situations where there is a contraindication to traditional anti-angina medications, or a concern about decreases in blood pressure or heart rate), or as add-on therapy to nitrates, $\beta$-blockers and calcium channel blockers [18]. Currently, ranolazine is indicated for patients with chronic stable angina, not for patients with 
Fig. 3 Patient-reported impact of angina on daily activities before and after taking ranolazine. $N=92$ respondents. The impact of angina on daily activities was rated using a 7-point Likert scale (where $1=$ not at all and $7=\mathrm{a}$ lot $)$

Fig. 4 Patient-reported changes in quality of life on the Patient Global Impression of Change scale since initiating ranolazine treatment. $N=92$ respondents
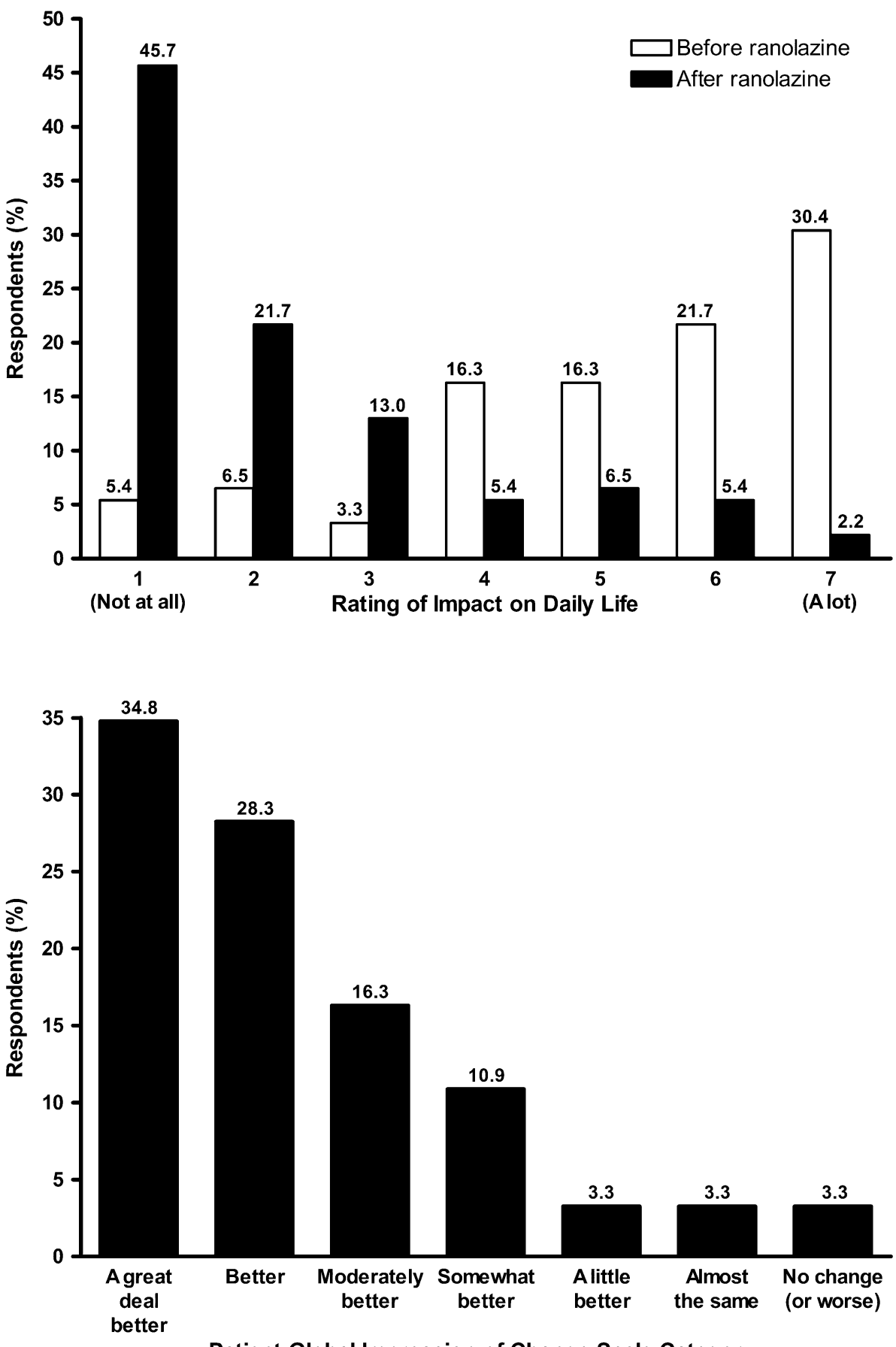

Patient Global Impression of Change Scale Category stable ischemic heart disease. However, some suggest that there is a need for ranolazine in the broader CHD population, such as in those with cardiac $\mathrm{X}$ syndrome, who often have no response to conventional anti-anginal therapy, or those with ischemic heart disease plus diabetes mellitus or arrthymias [19, 20]. While the high cost of ranolazine versus other anti-angina medications often leads to physicians opting to use ranolazine as a second-line or later treatment [18], the use of ranolazine in patients with poorly controlled angina is associated with decreases in revascularization rates, prescription costs, and a reduction in total care costs compared with patients receiving nitrates, $\beta$-blockers or calcium channel blockers [21]. Thus, the use of ranolazine can reduce the large financial burden chronic stable angina puts on the healthcare system.

The improvements in QoL and severity of angina attacks reported by respondents on ranolazine in the present survey reflect the efficacy of outcomes tools such as the SAQ used to assess QoL in patients with chronic stable angina [13]. The trend in improved QoL reported by respondents taking 
ranolazine in this survey mirror similar trends reported in randomized clinical trials in which ranolazine therapy was reported to improve angina and QoL scores in patients with acute coronary syndromes who had a history of chronic angina. Post-hoc analysis of QoL data from MERLINTIMI 36 indicated that the benefit of ranolazine was most apparent in the subgroup of patients with a history of prior angina (approximately $54 \%$ of the entire MERLIN population). Among these patients, significant effects versus placebo were seen on most domains assessed, with the greatest mean treatment effects observed for the SAQ assessments of angina frequency (mean treatment effect 3.4 points; $p<0.001)$, QoL (2.7 points; $p<0.001)$, and treatment satisfaction (1.5 points; $p=0.004)$ [11]. In addition, the results of a study in women with angina and myocardial ischemia showed that treatment with ranolazine produced significantly better median SAQ scores for physical functioning, angina stability, and QoL than placebo [10], and a study in a group of veterans with chronic stable angina who received ranolazine in addition to optimal doses of conventional therapy demonstrated clinically significant improvements from baseline in SAQ scores in the domains of physical limitation, angina stability, and disease perception after 1 and 3 months of treatment [22]. The survey results may also reflect the good tolerability of ranolazine in the appropriate patient subset when used over an extended duration (up to 4 years).

The present study has some limitations that should be considered when drawing conclusions. A control group was not established for comparative purposes, as only patients receiving ranolazine were recruited to participate. Nevertheless, as coronary artery disease is a gradually progressive disease, improvement from pretreatment values (while on background therapy) suggests a beneficial role for ranolazine. We could not account for confounding factors, and no information on the CHD profiles of the patients (i.e., the presence of obstructive/non-obstructive disease or normal arteries) was collected. The survey participants comprise a select group of respondents who were taking ranolazine and filling ranolazine prescriptions over time. Presumably, patients who did not respond to ranolazine would not have continued their participation in the panel; the proportion of patients who terminated ranolazine treatment and their reasons for doing so (e.g., efficacy, tolerability, expense) are unknown, although placebo-controlled study data give an indication of the proportion of patients who are anticipated to respond to ranolazine [23]. Given that only $54 \%$ of the total panel completed the survey, it is possible that some bias was introduced, because of potential differences between those who answered the survey and those who did not; however, patients were not pre-selected for a positive response to ranolazine, and in our experience, patients with a negative therapy experience are much more likely to respond to a survey than those with a positive experience, and thus we consider our results to be valid. While the accuracy of symptom recall over a relatively long period of time (6 months to 4 years) is a potential concern, the anginarelated impact on QoL was such that most patients felt comfortable assessing their symptoms; those who could not accurately recall or assess their symptoms were not recruited to the study. In addition, there was no difference in the results between those with 6 months' experience and those with 4 years' worth, which suggests that patient recall was reliable in this case. It should also be noted the use of the PGIC scale versus other validated scales for angina severity and QoL is an additional limitation; however, the SAQ was not included in this survey due to limitations associated with its length.

\section{Conclusion}

Patients with chronic angina maintaining treatment with ranolazine over time, with treatment durations ranging from $<6$ months to $>4$ years, reported substantial reductions in the severity and frequency of angina attacks, reductions in the impact of angina on daily activities, and improvements in QoL. These observations correspond to key treatment goals established by ACC/AHA guidelines for patients with stable ischemic heart disease.

Acknowledgments Funding for the patient survey was provided by Gilead Sciences, Inc. Luana Atherly, PhD, Scarlett Geunes-Boyer, $\mathrm{PhD}$, and Sushma Soni of inScience Communications, Springer Healthcare, provided medical writing support which was funded by Gilead Sciences, Inc.

Conflict of interest Dr. Grehan is currently a Gilead employee and owns Gilead stock and options. Dr. Muhlestein has received $<\$ 2,000$ dollars honorarium for consulting fees from Gilead Sciences, Inc.

Open Access This article is distributed under the terms of the Creative Commons Attribution Noncommercial License which permits any noncommercial use, distribution, and reproduction in any medium, provided the original author(s) and the source are credited.

\section{References}

1. Go AS, Mozaffarian D, Roger VL, et al. Heart disease and stroke statistics-2013 update: a report from the American Heart Association. Circulation. 2013;127(1):e6-245.

2. Brorsson B, Bernstein SJ, Brook RH, Werko L, for the SECOR/ SBU Project Group. Quality of life of patients with chronic stable angina before and four years after coronary revascularisation compared with a normal population. Heart. 2002;87(2):140-5.

3. Pragodpol P, Ryan C. Critical review of factors predicting healthrelated quality of life in newly diagnosed coronary artery disease patients. J Cardiovasc Nurs. 2013;28(3):277-84. 
4. Javitz HS, Ward MM, Watson JB, Jaana M. Cost of illness of chronic angina. Am J Manag Care. 2004;10(11 Suppl.):S358-69.

5. Beltrame JF, Weekes AJ, Morgan C, Tavella R, Spertus JA. The prevalence of weekly angina among patients with chronic stable angina in primary care practices: The Coronary Artery Disease in General Practice (CADENCE) Study. Arch Intern Med. 2009;169(16):1491-9.

6. Fihn SD, Gardin JM, Abrams J, et al. American College of Cardiology Foundation/American Heart Association Task Force. 2012 ACCF/AHA/ACP/AATS/PCNA/SCAI/STS guideline for the diagnosis and management of patients with stable ischemic heart disease: a report of the American College of Cardiology Foundation/American Heart Association Task Force on Practice Guidelines, and the American College of Physicians, American Association for Thoracic Surgery, Preventive Cardiovascular Nurses Association, Society for Cardiovascular Angiography and Interventions, and Society of Thoracic Surgeons. Circulation. 2012;126(25):e354-471.

7. Atmakuri SR, Gollob MH, Kleiman NS. Stable Angina. In: Rosendorff $\mathrm{C}$, editor. Essential cardiology: principles and practice. 2nd ed. Totowa: Humana Press; 2005. p. 451-70.

8. Ranexa [package insert]. Foster City, CA; Gilead Sciences, Inc.; 2011.

9. Chaitman BR, Pepine CJ, Parker JO, for the Combination Assessment of Ranolazine In Stable Angina (CARISA) Investigators, et al. Effects of ranolazine with atenolol, amlodipine, or diltiazem on exercise tolerance and angina frequency in patients with severe chronic angina: a randomized controlled trial. JAMA. 2004;291(3):309-16.

10. Mehta PK, Goykhman P, Thomson LEJ, et al. Ranolazine improves angina in women with evidence of myocardial ischemia but no obstructive coronary artery disease. JACC Cardiovasc Imaging. 2011;4(5):514-22.

11. Arnold SV, Morrow DA, Wang K, et al. Effects of ranolazine on disease-specific health status and quality of life among patients with acute coronary syndromes: results from the MERLIN-TIMI 36 randomized trial. Circ Cardiovasc Qual Outcomes. 2008;1(2):107-15.

12. Guy W. ECDEU Assessment Manual For Psychopharmacology, DHEW Publication No. ADM 76-338. Washington, DC: US Government Printing Office; 1976.
13. Spertus JA, Jones P, McDonell M, Fan V, Fihn SD. Health status predicts long-term outcome in outpatients with coronary disease. Circulation. 2002;106(1):43-9.

14. Venkitachalam L, Kip KE, Mulukutla SR, et al. for the NHLBIsponsored Dynamic Registry Investigators. Temporal trends in patient-reported angina at one year after percutaneous coronary revascularization in the stent era: a report from the NHLBIsponsored 1997-2006 dynamic registry. Circ Cardiovasc Qual Outcomes. 2009;2(6):607-15.

15. Weintraub WS, Spertus JA, Kolm P, for the COURAGE Trial Research Group, et al. Effect of PCI on quality of life in patients with stable coronary disease. N Engl J Med. 2008;359(7):677-87.

16. Boden WE, O'Rourke RA, Teo KK, for the COURAGE Trial Research Group, et al. Optimal medical therapy with or without PCI for stable coronary disease. N Engl J Med. 2007;356(15): 1503-16.

17. Pocock SJ, Henderson RA, Clayton T, Lyman GH, Chamberlain DA, for the RITA-2 Trial Participants. Quality of life after coronary angioplasty or continued medical treatment for angina: three-year follow-up in the RITA-2 trial. J Am Coll Cardiol. 2000;35(4):907-14.

18. Thadani U. Should ranolazine be used for all patients with ischemic heart disease or only for symptomatic patients with stable angina or for those with refractory angina pectoris? A critical appraisal. Expert Opin Pharmacother. 2012;13(17): 2555-63.

19. Cocco G. Management of myocardial ischemia. Is ranolazine needed? For all or some patients with myocardial ischemia? Expert Opin Pharmacother. 2012;13(17):2429-32.

20. Cocco G. Indicated and off-label use of ranolazine. E J Cardiol Pract. 2013;11(18).

21. Phelps CE, Buysman EK, Gomez Rey G. Costs and clinical outcomes associated with use of ranolazine for treatment of angina. Clin Ther. 2012;34(6):1395-407 e4.

22. Reeder DN, Gillette MA, Franck AJ, Frohnapple DJ. Clinical experience with ranolazine in a veteran population with chronic stable angina. Ann Pharmacother. 2012;46(1):42-50.

23. Cocco G, Rousseau MF, Bouvy T, et al. Effects of a new metabolic modulator, ranolazine, on exercise tolerance in angina pectoris patients treated with beta-blocker or diltiazem. J Cardiovasc Pharmacol. 1992;20(1):131-8. 